\title{
Electrically controllable liquid crystal photonic bandgap fiber with dual-frequency control
}

Scolari, Lara; Alkeskjold, Thomas Tanggaard; Riishede, Jesper; Nielsen, Martin Dybendal; Hermann, David Sparre; Anawati, A.; Bassi, Paolo; Bjarklev, Anders Overgaard

\author{
Published in: \\ IEEE Proceedings
}

Link to article, DOI:

10.1109/WFOPC.2005.1462093

Publication date:

2005

Document Version

Publisher's PDF, also known as Version of record

Link back to DTU Orbit

Citation (APA):

Scolari, L., Alkeskjold, T. T., Riishede, J., Nielsen, M. D., Hermann, D. S., Anawati, A., Bassi, P., \& Bjarklev, A. O. (2005). Electrically controllable liquid crystal photonic bandgap fiber with dual-frequency control. In IEEE Proceedings: Workshop on Fibres and Optical Passive Components (pp. 14-19). IEEE.

https://doi.org/10.1109/WFOPC.2005.1462093

\section{General rights}

Copyright and moral rights for the publications made accessible in the public portal are retained by the authors and/or other copyright owners and it is a condition of accessing publications that users recognise and abide by the legal requirements associated with these rights.

- Users may download and print one copy of any publication from the public portal for the purpose of private study or research.

- You may not further distribute the material or use it for any profit-making activity or commercial gain

- You may freely distribute the URL identifying the publication in the public portal 


\title{
Electrically Controllable Liquid Crystal Photonic Bandgap Fiber with Dual-Frequency Control
}

\author{
L. Scolari ${ }^{1,2}$, T. T. Alkeskjold ${ }^{1}$, J. Riishede ${ }^{1}$, M. D. Nielsen ${ }^{3}$, D. S. Hermann ${ }^{4}$, A. Anawati ${ }^{4}$, \\ P. Bassi ${ }^{2}$, and A. Bjarklev ${ }^{1}$ \\ ${ }^{1}$ Research Center COM, Technical University of Denmark, DK-2800 Lyngby, Denmark \\ lscolari@deis.unibo.it ttl@com.dtu.dk \\ 2 Dipartimento di Elettronica, Informatica e Sistemistica, University of Bologna, Viale Risorgimento 2, \\ I 40136, Bologna, Italy \\ ${ }^{3}$ Crystal Fibre A/S, Blokken 84, DK-3460 Birkerød, Denmark. \\ ${ }^{4}$ Photonics Laboratory, Department of Microtechnology and Nanoscience MC2, \\ Chalmers University of Technology, 41296 Gothenburg, Sweden.
}

\begin{abstract}
We present an electrically tunable liquid crystal photonic bandgap fiber device based on a dual frequency liquid crystal with pre-tilted molecules that allows the bandgaps to be continuously tuned. The frequency dependent behavior of the liquid crystal enables active shifting of the bandgaps toward shorter or longer wavelengths. We also describe and experimentally characterize an electro-optical modulator and a birefringence controller suitable for polarization control.
\end{abstract}

\section{INTRODUCTION}

Photonic crystal fibers (PCFs) are microstructured waveguides with a large number of air holes usually located in the cladding region. The microstructured refractive index profile in the fiber cross section allows light guidance by the so called modified Total Internal Reflection (m-TIR) or by the Photonic Bandgap (PBG) effect [1,2]. Optical properties of these fibers can be manipulated by filling the air holes with liquid materials. In [3] a device with tunable transmission properties was demonstrated by filling the air holes with polymers. In [4] the air holes of a PCF were infiltrated with a high index oil and a tunable photonic bandgap fiber was demonstrated. In $[5,6]$ it was shown that Liquid Crystal (LC) filled PCF can form tunable photonic bandgap waveguides. Tunability was achieved by taking advantage of the thermo-optic effect to induce index changes. Then electrical control of a nematic LC filled PCF (LCPCF) has been proposed and demonstrated [7,8]. In [7] an ordinary nematic LC was used to infiltrate the air holes of a solid-core

L. Scolari was supported by a fellowship of the Faculty of Engineering of the University of Bologna.
PCF. This transforms the structure changing the m-TIR fiber into a PBG one. The device was then placed between two electrodes. The applied electric field causes a reorientation of the LC molecules which induces a change in the refractive index of the LC. This LCPCF has been used as an optical switch with rise and decay times of 5 and $52 \mathrm{~ms}$, respectively. However, such a device did not allow accurate and continuous control of the spectral position of the bandgaps because of the presence of reverse tilt domain defects in the LC and electric field threshold effects due to the planar alignment of the nematic LC. Here we present an electrically tunable LCPCF device based on a dual frequency nematic LC. This new device does not suffer from both the problems presented by the former one, thereby allowing the bandgaps to be continuously tuned. In the next sections the characteristics of our device will be described and experimental results concerning the continuous tunability and the frequency dependent behavior of the LCPCF will be illustrated. Two possible applications will be presented at the end of the article: an electro-optical modulator and a birefringence controller with $\mathrm{ms}$ response times.

\section{THE LCPCF FIBER DEVICE}

The fiber used in the experiment is a so called 'Large Mode Area' PCF with a solid silica core surrounded by 5 rings of air holes arranged in a triangular lattice, as shown in figure 1 . The hole size $(d)$ is $5 \mu \mathrm{m}$, the inter-hole distance $(\Lambda)$ is $10 \mu \mathrm{m}$ and the fiber diameter $(D)$ is $125 \mu \mathrm{m}$. This fiber guides light by the principle of $\mathrm{m}$-TIR. The PCF is filled for $8 \mathrm{~mm}$ with a dual frequency nematic LC (Merck, Darmstadt, Germany, MDA-003969) using capillary forces. The LC is then positioned $2 \mathrm{~mm}$ 
further into the fiber using a high-pressure chamber and the LCPCF is spliced to a PCF of the same type. The splice loss is approximately $0.3 \mathrm{~dB}$ and the insertion loss of the device is approximately $6 \mathrm{~dB}$ at $\lambda=1600 \mathrm{~nm}$, including the splice loss. The section of the PCF containing LC is placed between two electrodes.

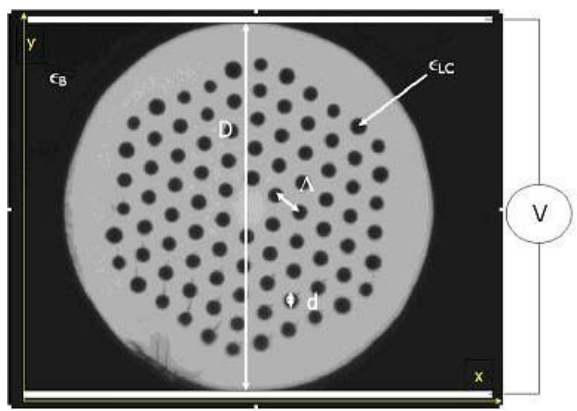

Fig.1 Micrograph of the end facet of a LCPCF sandwiched between two electrodes. Its most important parameters are illustrated.

The dual frequency LC used in the experiment is an uniaxial birefringent medium with ordinary and extraordinary refractive indices $n_{o}=1.4978$ and $n_{e}=1.7192$ respectively, at $\lambda=589.3 \mathrm{~nm}$. At the frequency $\mathrm{f}=1 \mathrm{kHz}$, the values of the $\mathrm{LC}$ permittivity along the ordinary and extraordinary axes are $\varepsilon_{\mathrm{o}}=7.3 \varepsilon_{0}$ and $\varepsilon_{\mathrm{e}}=$ $10.6 \varepsilon_{0}$, respectively. The most interesting property of this LC is that the dielectric anisotropy $\Delta \varepsilon$, defined as $\varepsilon_{\mathrm{e}}-\varepsilon_{\mathrm{o}}$, becomes negative for increasing frequency, as shown in figure 2 . In particular, $\Delta \varepsilon=2.99$ at $\mathrm{f}=1 \mathrm{kHz}$ and $\Delta \varepsilon=-2.76$ at $\mathrm{f}=50 \mathrm{kHz}$.

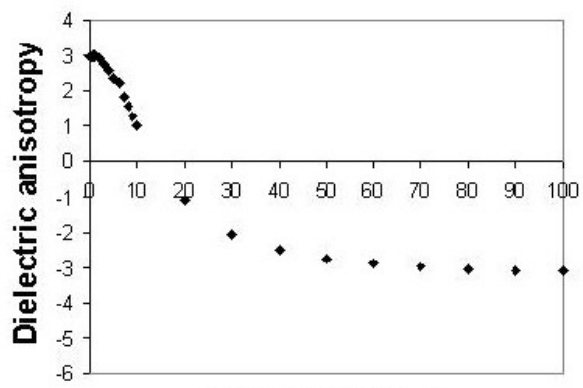

Frequency $(\mathrm{kHz})$

Fig.2 Dielectric anisotropy $\Delta \varepsilon$ as a function of the frequency of the E-field applied to the electrodes.

Polarization microscopy studies of a $5 \mu \mathrm{m}$ fused silica capillary tube infiltrated with LC shows that MDA-00-3969 is aligned in a splayed configuration with an alignment at the surface of about 45 degrees and with this angle opening up towards the opposite direction as the direction of filling, as illustrated in figure 3-b.
Polarized micrographs of the silica tube filled with MDA-003969 are shown in figure 3 -a. The top left picture is when the angles between the capillary axis and the crossed polarizers are 0 and 90 degrees and the bottom left picture is when the angle between the capillary and both the crossed polarizers is 45 degrees. The picture in the centre is intermediate between the former two cases.

The LC alignment illustrated in figure 3 is the key feature of the LCPCF device because it provides new characteristics respect to a device based on an ordinary nematic LC. In particular no reverse tilt domain defects and threshold effects are present due to the splayed alignment.
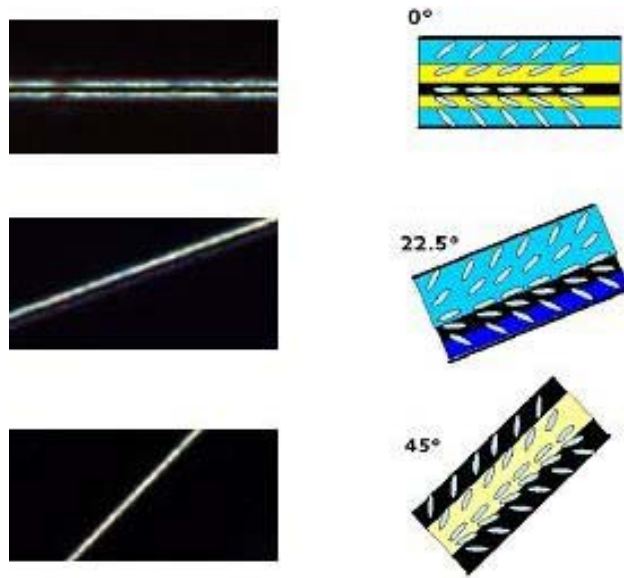

(a)

(b)

Fig.3 Polarized micrograph of a $5 \mu \mathrm{m}$ silica capillary infiltrated with a dual frequency LC (MDA-00-3969). Capillary is angled at 0 degrees (top), 22.5 degrees (centre) and 45 degrees (bottom).

\section{MANIPULATION OF SPECTRAL PROPERTIES}

A simple theoretical model can be used to understand the principles on which the tunability is based [9]. Let us remind that the infiltration of the air holes with the LC changes the waveguiding properties of the infiltrated section, since the refractive index of the core is lower than that of the encircling cladding. The m-TIR based guidance is not possible and the section in which the LC is infiltrated can support only a number of guided wavelength bands due to the anti-resonant reflection (bandgaps) from the LC filled holes. If the refractive index of the LC is somewhat higher than that of silica, the LC infiltrated holes can be approximated as isolated waveguides and the spectral position of the bandgaps can be determined using a simple cutoff approach. In the isotropic case, the transmission minima occur at wavelengths given by this simple analytical formula [9]: 


$$
\lambda_{m}=\frac{2 d}{m+1 / 2} \sqrt{n_{2}^{2}-n_{1}^{2}}
$$

where $m$ is an integer, $d$ is the hole size, $n_{2}$ the isotropic refractive index of high-index inclusions and $n_{1}$ the refractive index of the background material $\left(\mathrm{n}_{1} \approx 1.45\right.$ for silica, as in this case). This expression is only valid when the LC infiltrated holes can be approximated as isolated waveguides, or:

$$
\frac{\lambda}{d} \leq 2 \sqrt{n_{2}^{2}-n_{1}^{2}}
$$

Since the transmission minima depend of $\mathrm{n}_{2}$, the position of the transmission dips can be shifted simply tuning $\mathrm{n}_{2}$. This can be achieved using materials with electro-optic properties, as LCs. The molecules of the LC have a preferred direction along which they tend to be oriented. This direction is defined by a unit vector termed director. When an electric field is applied to the LC it will exert a torque on the director. Depending on the sign of the anisotropy, i.e. $\Delta \varepsilon>0$ or $\Delta \varepsilon<0$, this torque will turn the director respectively toward being parallel or perpendicular to the field direction, as illustrated in figure 4.

The refractive index $\mathrm{n}_{2}$ experienced by the light propagating in the LCPCF device changes depending on the E-field intensity.

The bandgaps shift is given by the following formula:

$$
\Delta \lambda_{m}=\frac{2 d}{m+1 / 2}\left(\sqrt{n_{2}^{2}\left(E_{o n}\right)-n_{1}^{2}}-\sqrt{n_{2}^{2}\left(E_{o f f}\right)-n_{1}^{2}}\right)
$$

where $n_{2}\left(E_{\text {on }}\right)$ is the refractive index of the LC when a voltage is applied and $\mathrm{n}_{2}\left(\mathrm{E}_{\text {off }}\right)$ is the refractive index of the $\mathrm{LC}$ when no voltage is applied.

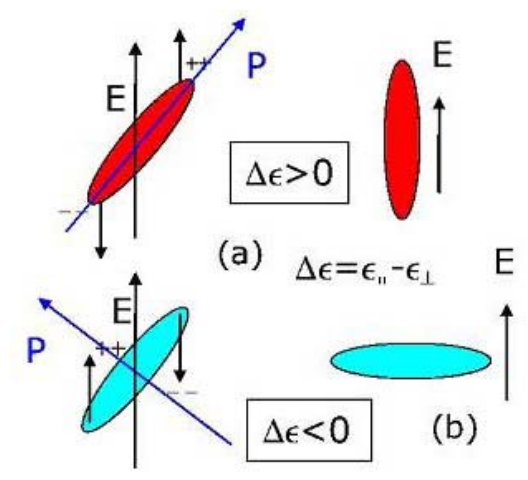

Fig.4 Depending on the sign of the dielectric permittivity, the induced polarization $\mathbf{P}$ gives a dielectric torque to the molecules, turning the director respectively toward being parallel (top) or perpendicular (bottom) to the field direction.

In the next section the most interesting properties of the LCPCF will be introduced: continuous tunability, active shift toward longer or shorter wavelengths, dynamic properties and ability for continuous polarization control.

\section{CONTINUOUS TUNABILITY OF THE BANDGAPS}

In this section, the experimental demonstration of continuous bandgap tunability will be described.

A $1 \mathrm{kHz}$ sine wave signal is applied to the electrodes using the experimental setup illustrated in figure 5 .

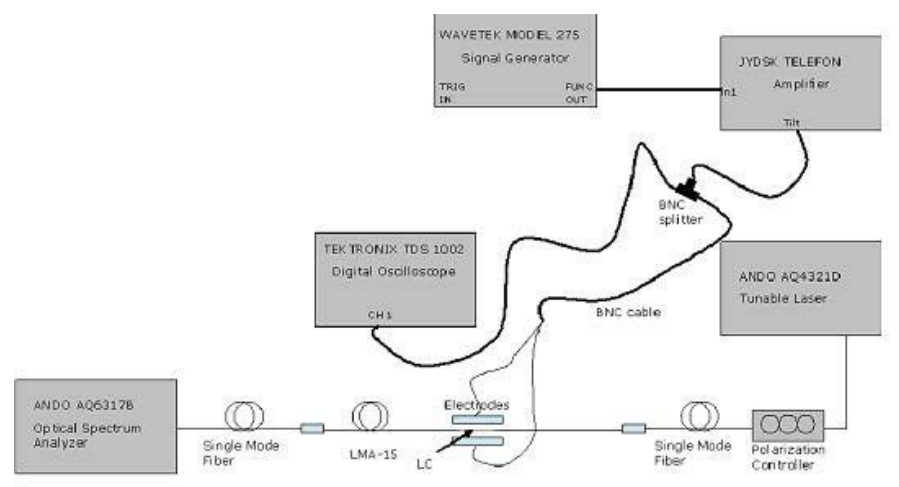

Fig. 5 Diagram of the experimental setup used to measure the shift of the bandgaps.

Polarized light with $\lambda$ in the range from 1520 to $1620 \mathrm{~nm}$ is launched in the LCPCF device. As the bandgaps shift is polarization dependent, the polarization controller is used to find the polarization that gives the maximum transmission. The voltage applied to the electrodes is increased until a small shift in the bandgaps is observed. When the voltage is about $19 \mathrm{~V}_{\mathrm{rms}}$ a shift of $0.3 \mathrm{~nm}$ is observed. Continuous tunability of the bandgaps is observed by further increasing of the applied voltage, as shown in figure 6 .

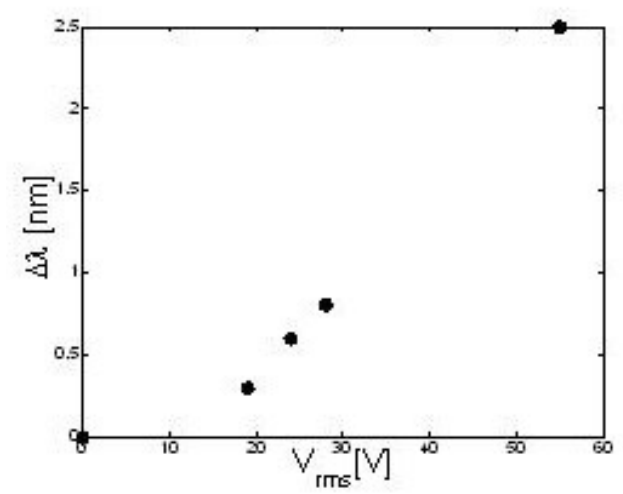

Fig. 6 Positive shift of the bandgaps for different voltages when a $1 \mathrm{kHz}$ E-field is applied to the LCPCF.

Continuous tunability is caused by the splayed alignment of the 
LC. The LC mesogens near the surface start to rotate for value of the electric field below the threshold required for the mesogens that lie in the centre of the capillary. Therefore, the change of the refractive index $\mathrm{n}_{2}$ is continuous and, as a consequence of it, the shift is highly controllable. This kind of behavior is not present in ordinary nematic LCPCFs as all the mesogens are equally oriented with respect to the field and the change of the refractive index $n_{2}$ is abrupt due to the Fredericks transition [10].

Moreover, the frequency dependent behavior of the LC enables active shifting toward longer or shorter wavelengths depending on the frequency of the applied electric field, as shown in figure 7.

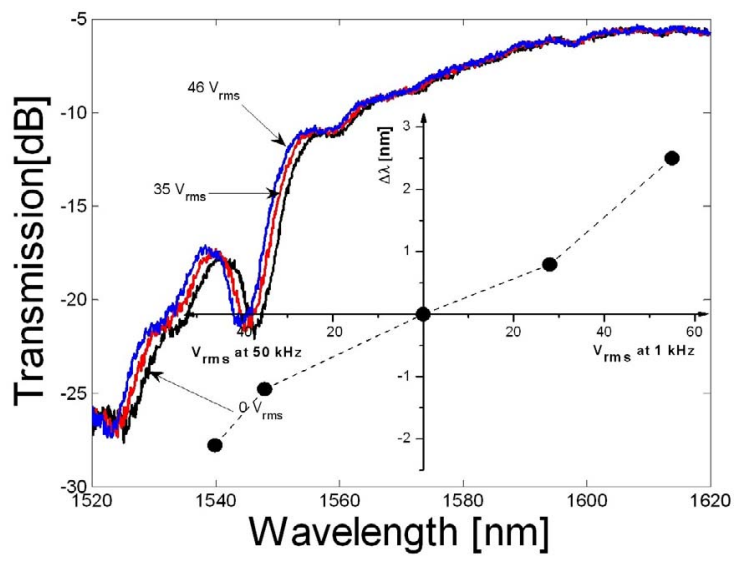

Fig.7 Negative shift of the bandgaps when the frequency of the applied electric field is $50 \mathrm{kHz}$. The inset shows the dual frequency behavior of the device: the shift is negative (toward shorter wavelengths) when the frequency of the electric field is $50 \mathrm{kHz}$ and positive (toward longer wavelengths) at $1 \mathrm{kHz}$.

This dual frequency behavior can be well understood if we consider the alignment of MDA-00-3969 and we apply (3). As illustrated in figure 8 -a, at $\mathrm{f}=1 \mathrm{kHz}$, as $\Delta \varepsilon>0$, then the $\mathrm{LC}$ tends to lie along the field direction and, consequently, $\mathrm{n}_{2}\left(\mathrm{E}_{\mathrm{on}}\right)>\mathrm{n}_{2}\left(\mathrm{E}_{\mathrm{off}}\right)$. Using (3) one can see that the shift $\Delta \lambda_{\mathrm{m}}$ is positive. At $\mathrm{f}=50$ $\mathrm{kHz}$, as $\Delta \varepsilon<0$, then $\mathrm{n}_{2}\left(\mathrm{E}_{\mathrm{on}}\right)<\mathrm{n}_{2}\left(\mathrm{E}_{\text {off }}\right)$ and the shift $\Delta \lambda_{\mathrm{m}}$ is negative.

\section{SWITCHING PROPERTIES OF THE DEVICE}

Switching properties of the device are investigated as a function of the applied voltage by coupling polarized light at $1551 \mathrm{~nm}$ into the LCPCF device. The transmitted light is then detected by using a photodiode and an oscilloscope. The chosen wavelength is located on the low-wavelength bandgap edge whereby the light can be amplitude modulated by shifting the bandgaps. When a 1 $\mathrm{kHz}$ E-field is applied to the electrodes, the bandgaps shift toward longer wavelengths and the transmission at $1551 \mathrm{~nm}$ is lowered. On the contrary, when no field is applied to the LCPCF device, the transmission at $1551 \mathrm{~nm}$ is higher. Therefore, to obtain an electro-optical modulator, a $1 \mathrm{kHz}$ sine wave is modulated by a $50 \%$ duty cycle $10 \mathrm{~Hz}$ square wave signal and applied to the electrodes.

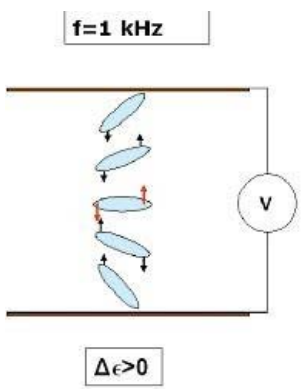

(a)

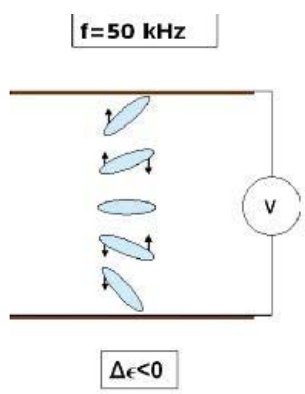

(b)
Fig. 8 Reorientation of the $\mathrm{LC}$ when a $1 \mathrm{kHz}$ voltage (a) and a $50 \mathrm{kHz}$ voltage (b) are applied to the LC MDA-00-3969.

As the bandgaps are polarization sensitive, the polarization of the $1551 \mathrm{~nm}$ light is adjusted for maximum transmission. The light detected by the photodiode is amplitude modulated, as illustrated in figure 9 where the photodiode voltage has been shown.

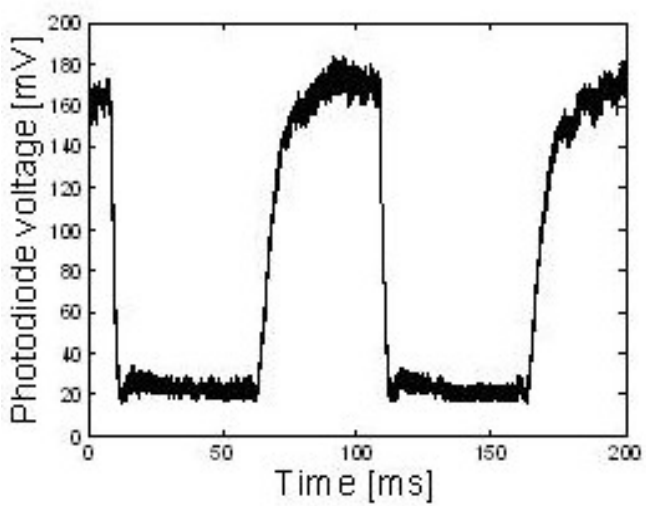

Fig. 9 Photodiode voltage when a $1 \mathrm{kHz}$ sine wave with a $96 \mathrm{~V}_{\mathrm{rms}}$ voltage and amplitude modulated by a $10 \mathrm{~Hz}$ square signal is applied to the electrodes.

Different voltages are applied to the electrodes. The rise and decay time $\tau_{\mathrm{ON}}$ and $\tau_{\mathrm{OFF}}$ are measured from $10 \%$ to $90 \%$ amplitude modulation and are illustrated in figure 10 as a function of the applied voltage. The rise time is the time from high to low transmission and the decay time is the time from low to high transmission. As shown, the response time is strongly limited by the decay time $\tau_{\mathrm{OFF}}$. 
We believe that the decay time can be comparable with the rise time if a frequency modulation scheme is used instead of an amplitude modulation scheme. The frequency modulation scheme should switch between a $1 \mathrm{kHz}$ signal and a $50 \mathrm{kHz}$ signal. When the $1 \mathrm{kHz}$ signal is applied, the LC mesogens are turned toward being parallel to the field. When the $50 \mathrm{kHz}$ signal is applied, then $\Delta \varepsilon<0$ and the dielectric torque helps the LC molecules to move back into the equilibrium. On the contrary, in the case of amplitude modulation there are not any dielectric torques that assist the LC molecules back to the equilibrium and the decay time is higher respect to the rise time.

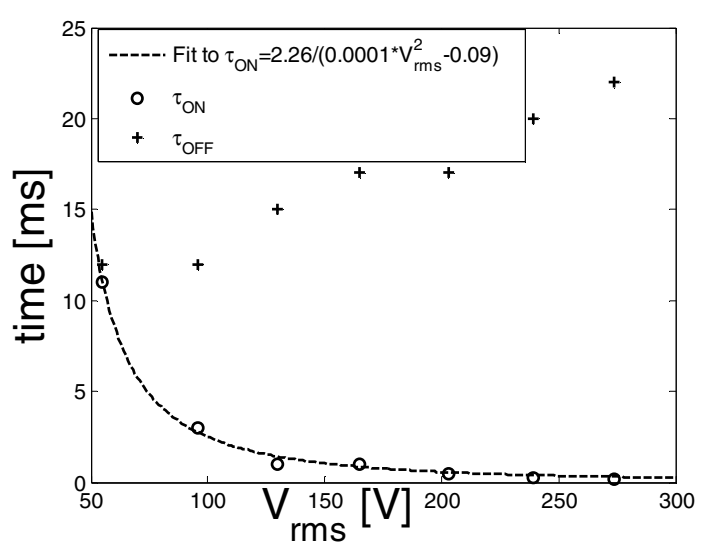

Figure 10 Measured rise and decay times of our LCPCF device as a function of the applied voltage.

\section{BIREFRINGENCE TUNABILITY}

A further feature offered by this device is the control of the birefringence. Since the LC is anisotropic, the LCPCF birefringence can be tuned when an E-field is applied because the guided modes losses their degeneracy and experience a different phase delay when propagating through the LCPCF.

This possibility of having electrical control of birefringence can find use in electrically controlled polarization controllers. Birefringence control has also been demonstrated in [11] where some of the air holes of a 'grapefruit' fiber were infiltrated with polymer and tunability was achieved by temperature tuning the polymer refractive index.

A polarized laser source at $1600 \mathrm{~nm}$ was connected to the LCPCF through a polarization analyzer, as shown in figure 11. The polarization analyzer launches the light in the LCPCF and resolves the output power into the Stokes parameters, plotted on the Poincaré sphere. The Poincaré sphere maps all the states of polarization to the surface of a sphere. Each point on the sphere represents a unique polarization state. The north and the south poles represent circularly polarized light. Points on the equator indicate linear polarization. Other points on the sphere represent elliptical polarization.

When an electric field is applied to the LCPCF, the LC reorients depending on the applied voltage. The two orthogonal polarized guided modes experience different refractive indices compared to the case in which the field is off and this introduces a phase shift between them. Any change in phase shift between the two orthogonal polarizations corresponds to a rotation on the Poincaré sphere.

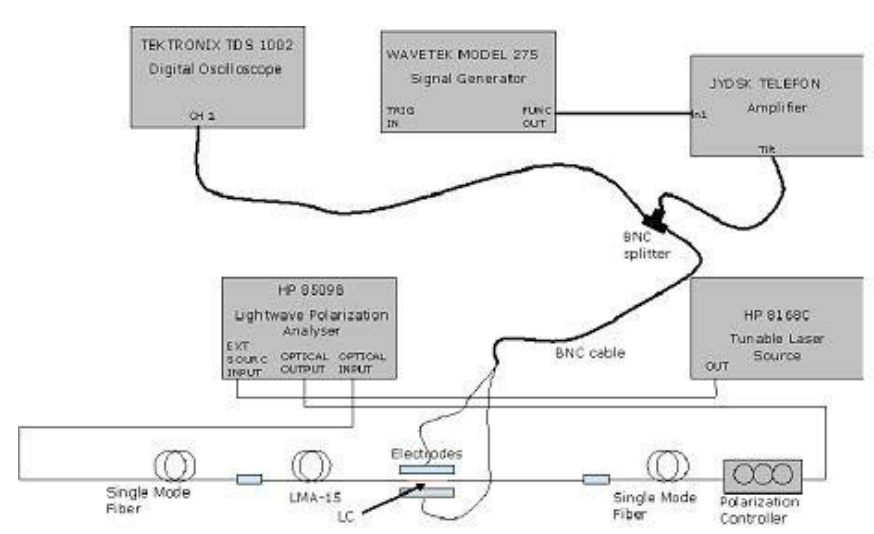

Fig. 11 Diagram of the setup used to investigate the relative birefringence as a function of the applied electric field

The birefringence can be deduced from the phase by the following formula:

$$
\phi=\left(\beta_{x}-\beta_{y}\right) L=2 \pi\left(n_{x}-n_{y}\right) L / \lambda=2 \pi \Delta n L / \lambda
$$

where $\mathrm{L}$ is the length of the PCF containing the $\mathrm{LC}$ and $\beta_{x}$ and $\beta_{y}$ are the propagation constant related to the effective indices $n_{x}$ and $n_{y}$, respectively.

If an electric field is applied to the LCPCF, the phase shift is related to the change in birefringence by the following:

$$
\Delta \phi=2 \pi\left(\Delta n-\Delta n^{\prime}\right) L / \lambda
$$

where $\Delta n$ and $\Delta n '$ is the birefringence with and without an applied electric field, respectively. Therefore, the change in birefringence caused by the application of a voltage can be determined from the phase shift through this formula:

$$
\Delta n-\Delta n^{\prime}=\frac{\Delta \phi \lambda}{2 \pi L}
$$

Different voltages were applied to the LCPCF: $18 \mathrm{~V}_{\mathrm{rms}}, 35 \mathrm{~V}_{\mathrm{rms}}$, $55 \mathrm{~V}_{\text {rms }}, 82 \mathrm{~V}_{\text {rms. }}$. The phase shift $\Delta \phi$ has been measured using the Stokes parameters plotted on the Poincaré sphere and the relative change in birefringence caused by the applied electric field has been calculated using formula (7). The results are shown in 
figure 13 .

These experimental results demonstrate that, as the birefringence is a function of the strength of the applied electric field, our LCPCF device can find a new application working as a polarization controller with $\mathrm{ms}$ response times.

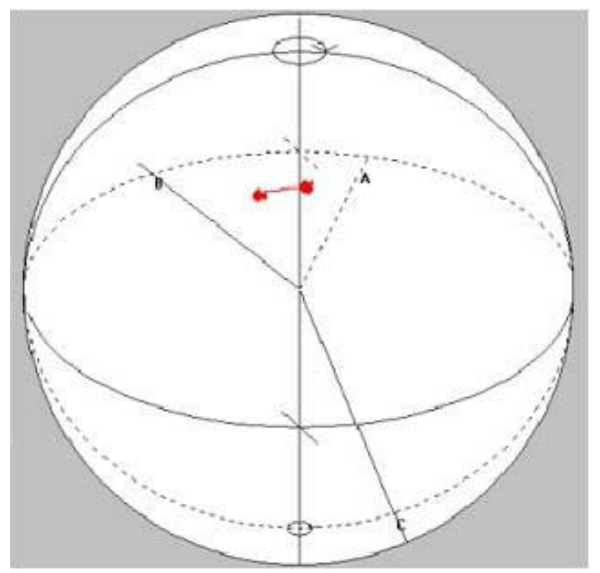

Fig. 12 Phase shift on the Poincare sphere when a $35 \mathrm{~V}_{\text {rms }}$ voltage is applied to the LCPCF device.

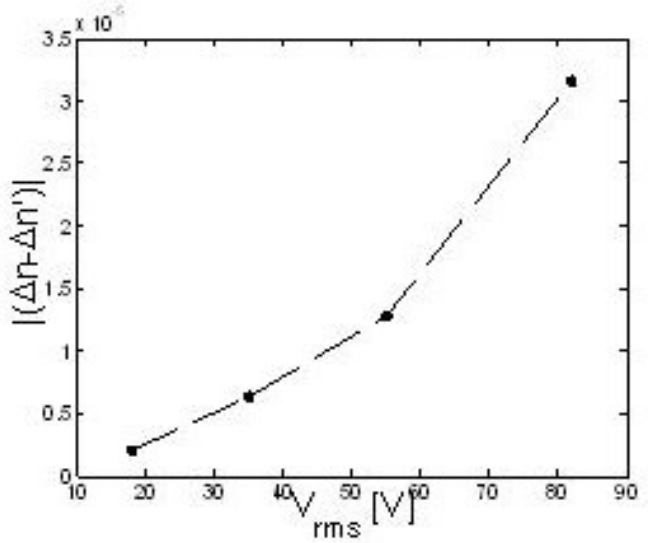

Fig. 13 Plot of the relative change in birefringence as a function of the applied voltage.

\section{CONCLUSION}

An electrically tunable LCPCF that allows accurate and continuous tunability of the spectral position of the bandgaps has been demonstrated. Continuous control is achieved by filling the air holes with the dual frequency LC MDA-00-3969 from Merck, Germany. The alignment of this LC is not planar and, consequently, do not present reverse tilt domain defects or threshold effects that do not allow a continuous tunability, as found by previous demonstrations. An electro-optical modulator and a birefringence controller have been presented and experimentally characterized. The rise and decay times have been measured and improved respect to previous demonstrations. A further improvement can be obtain by using a frequency modulation scheme instead of an amplitude modulation scheme. Possible applications of these electrically tunable devices are polarization controllers, tunable attenuators, electro-optical modulators and tunable optical filters.

\section{ACKNOWLEDGMENT}

The authors would like to thank M. W. Haakestad (Department of Electronics and Telecommunications, Norwegian University of Science and Technology, N-7034 Trondheim, Norway) for helpful suggestions.

\section{REFERENCES}

[1] P. Russell, "Photonic crystal fibers," Science 299, 358-362 (2003)

[2] J. C. Knight, J. Broeng, T. A. Birks, and P. St. J. Russell, "Photonic band gap guidance in optical fibers," Science 283, 1476-1478 (1998)

[3] B. J. Eggleton, C. Kerbage, P. S. Westbrook, R. Windeler and A. Hale, "Microstructured optical fiber devices, " Opt. Express 9, 698-713 (2001), http://www.opticsexpress.org/abstract.cfm?URI=OPEX-9-13-698

[4] R. T. Bise, R. S. Windeler, K. S. Kranz, C. Kerbage, B. J. Eggleton, and D. J. Trevor, "Tunable photonic band gap fiber" in OSA Trends in Optics and Photonics (TOPS) 70, Optical Fiber Communication Conference Technical Digest, Postconference Edition (Optical Society of America, Washington, DC, 2002) 466-468

[5] T. T. Larsen, A. Bjarklev, D. S. Hermann, and J. Broeng, "Optical devices based on liquid crystal photonic bandgap fibers," Opt. Express 11, 25892596 (2003), http://www.opticsexpress.org/abstract.cfm?URI=OPEX-1120-2589

[6] T. T. Alkeskjold, J. Lægsgaard, A. Bjarklev, D. S. Hermann, Anawati, J. Broeng, Jun Li and Shin-Tson Wu, "All-optical modulation in dye-doped nematic liquid crystal photonic bandgap fibers," Opt. Express 12, 5857 5871 (2004), http://www.opticsexpress.org/abstract.cfm?URI=OPEX-1224-5857

[7] M. W. Haakestad, T. T. Alkeskjold, M. D. Nielsen, L. Scolari, J. Riishede, H. E. Engan and A. Bjarklev, "Electrically Tunable Photonic Bandgap Guidance in a Liquid Crystal Filled Photonic Crystal Fiber," IEEE Phot. Tech. Lett. 17, 819-821 (2005)

[8] F. Du, Y.-Q. Lu and S.-T. Wu, "Electrically tunable liquid-crystal photonic crystal fiber," Appl. Phys. Lett. 85, 2181-2183 (2004)

[9] N. M. Litchinitser, S. C. Dunn, P. E. Steinvurzel, B. J. Eggleton, T. P. White, R. C. McPhedran and C. M. de Sterke, "Application of an ARROW model for designing tunable photonic devices," Opt. Express 12, 15401550 (2004) http://www.opticsexpress.org/abstract.cfm?URI=OPEX-12-8 $1540 \% 20 \% 20$

[10] V. G. Chigrinov, Liquid Crystal Devices, (Artech House, 1999)

[11] C. Kerbage and B. J. Eggleton, "Numerical analysis and experimental design of tunable birefringence in microstructured optical fiber," Opt. Express 10, 246-255 (2002) http://www.opticsexpress.org/abstract.cfm?URI=OPEX-10-5-246 